\title{
Troponin Levels Relate to CRP Concentrations in Patients With NAFLD on Maintenance Haemodialysis: A Retrospective Study
}

\author{
Domenico Capone - Mauro Vinciguerra - Annalisa Ragosta • \\ Vincenzo Citro · Giovanni Tarantino (D)
}

Received: March 12, 2020 / Published online: June 8, 2020

(C) The Author(s) 2020

\begin{abstract}
Introduction: Several studies have shown that high sensitivity cardiac troponin (hs-cTnT) levels are elevated in patients suffering from end-stage renal disease (ESRD), even in the absence of clinical signs and instrumental features of symptomatic acute coronary syndrome (ACS). In patients undergoing haemodialysis because of ESRD, nephrologists bear witness to this increase, whose origin and clinical impact are not yet well defined.
\end{abstract}

Digital Features To view digital features for this article go to https://doi.org/10.6084/m9.figshare.12206234.

\section{Capone}

Integrated Care Department of Public Health and Drug-Use, Section of Medical Pharmacology and

Toxicology, "Federico II" University, Naples, Italy

M. Vinciguerra

Section of Nephrology, Santa Maria Delle Grazie Hospital, Pozzuoli, Italy

\section{A. Ragosta}

Outpatients Clinic of Hemodialysis Dial Center s.r.l. Pomigliano D'Arco, Naples, Italy

V. Citro

Department of General Medicine, Umberto I"

Hospital, Nocera Inferiore, SA, Italy

G. Tarantino $(\square)$

Department of Clinical Medicine and Surgery,

"Federico II" University, Medical School, Naples, Italy

e-mail: tarantin@unina.it
Methods: By a retrospective study, we evaluated data from records of 70 patients with ESRD on haemodialysis, all of them with a history of NAFLD, not suffering for at least 3 months from symptomatic angina and without a history of ischemic heart disease in the same period.

Results: Hs-cTnT and C-reactive protein (CRP) levels both increased and were correlated, rho $=0.34, P=0.004$. The correlation coefficient between troponin and age was significant, rho $=0.47, P=0.0001$. Serum concentrations of hs-cTnT for the whole population were positively predicted by CRP levels, $P=0.004$. On separation of the population by gender, significant correlation between hs-cTnT and CRP was not found in women and was only present in men, $P=0.66$ and $P=0.000$, respectively.

Discussion: The assessment of hs-cTnT levels could represent a biological marker in particular subgroups of haemodialysis patients, especially for male patients with higher CRP, those at greater risk of silent myocardial ischemia and future major adverse cardiac events.

Conclusions: The evaluation of hs-cTnT in haemodialysed patients with NAFLD could indicate that men with higher CRP should undergo close monitoring in order to adopt specific therapy.

Keywords: C-reactive protein; End-stage renal disease; Gender; Haemodialysis; Hs-cardiac troponin; Non-alcoholic fatty liver disease; Silent myocardial ischemia 


\section{Key Points}

Why carry out this study?

Seventy patients on haemodialysis, with a history of non-alcoholic fatty liver disease (NAFLD), not suffering for at least 3 months from symptomatic angina and without a history of ischemic heart disease in the same period, were studied.

Troponin (hs-cTnT) levels were analysed to identify, among patients with higher C-reactive protein (CRP), those at greater cardiac risk.

We hypothesised that both CRP and troponin levels could be potential markers of silent ischemia.

\section{What was learned from the study?}

Both hs-cTnT and CRP concentrations in our population were increased.

The correlation coefficient between troponin and age was significant.

On analysis of the population by gender, significant correlation between hs-cTnT and CRP was not found in women and was present only in men.

Assessment of troponin levels could represent a biological marker in haemodialysis patients to identify those at greater risk of silent myocardial ischemia and future major adverse cardiac events. This would be of particular use in certain subgroups such as male patients with higher CRP.

This study could indicate which patients required stricter surveillance and more aggressive therapy with beta blockers and/ or ACE inhibitors.
As future directions, it could be interesting to evaluate in a prospective study the incidence of adverse cardiovascular events and all-cause mortality over time in the most inflamed patients with higher hscTnT levels according to the above hypothesis.

\section{INTRODUCTION}

Patients with non-alcoholic fatty liver disease (NAFLD) have a significantly higher risk of developing advanced chronic kidney diseases (CKD) when compared to patients without it [1]. Furthermore, a systematic review and metaanalysis of 34 studies showed that NAFLD was associated with an increased risk for coronary artery disease (HR 2.31) [2]. NAFLD has a strong independent association with cardiovascular (CV) events, even in an advanced CKD cohort with high comorbidity, implicating that routine screening for NAFLD may be warranted in CKD populations to enable targeted interventions for $\mathrm{CV}$ disease prevention in higher-risk patients [3]. Still, authors showed that carotid atherosclerosis is advanced in renal transplant recipients (RTR) with NAFLD, alerting to the existence of an increased CV risk in RTRs [4].

Cardiac troponins $\mathrm{T}$ and $\mathrm{I}$ are proteins responsible for regulating muscle contraction and are almost exclusively found at the cardiac level; for this reason they represent an important marker of myocardium necrosis and are used to diagnose acute cardiac damage [5].

Some studies have shown that high sensitivity cardiac troponin (hs-cTnT) levels are elevated in patients suffering from end-stage renal disease (ESRD), even in the absence of clinical signs and instrumental features of symptomatic acute coronary syndrome (ACS) [6, 7]. In patients undergoing haemodialysis because of ESRD, nephrologists confirm this increase, whose clinical impact is not yet well defined.

Hs-cTnT is widely used in everyday practice and is one of the cornerstones of the diagnosis of ACS in symptomatic patients presenting with angina at rest or triggered by physical activity. 
In patients with chronic renal failure undergoing haemodialysis and not opportunely treated for angina, assessing troponin levels is of particular importance to evaluate the efficacy of long-term therapy. A significant increase of the 3-h value undoubtedly leads to the diagnosis of ACS [8]. In haemodialysed patients, an increased troponin value, mainly if recurrent, is easily found in the absence of cardiac ischemia, even though the significance in this particular group of patients is not fully understood [9]. A fascinating hypothesis could be that the hscTnT levels represent a laboratory marker of an unrecognised or silent myocardial ischemia (SMI), as a consequence of non-renal causes, i.e. beyond the well-accepted renal failure [10]. In fact, both SMI and ventricular arrhythmias were noted most frequently during the last hour of dialysis [11]. In haemodialysed patients, dialysis catheters, the interaction of the patient's blood with the dialysis, endotoxaemia (from intestinal hypoperfusion or dialysis water) and more rapid loss of residual kidney function may contribute to inflammation [12]. Still, changes in the blood volume, electrolytes and osmolality would be expected to provide sufficient stimuli to induce haemodynamic modifications during a haemodialysis session [13]. Excessive renin secretion, increased arterial stiffness due to vascular calcifications, and increased activity of the sympathetic nervous system could induce blood pressure modifications that represent a high cardiac risk factor $[14,15]$.

The causes of inflammation in dialysis are multifactorial and include both dialysis-related and unrelated factors. Noteworthy, C-reactive protein (CRP) is a predictor of increased risk of onset of cardiac complications and therefore of mortality $[16,17]$. It has already been seen that patients with chronic renal insufficiency and in particular those under haemodialysis treatment generally have higher levels of CRP than similar populations without ESRD. Moreover, the higher the CRP levels are, the worst the prognosis [18]. Furthermore, although there is previous excellent research in the general population on detecting hs-cTnT as a major mortality risk [19], the role played by gender is not sufficiently documented and the role of age is somehow overlooked when analysing the link between the dialysis-associated inflammatory process and hs-cTnT levels.

Our aim was to evaluate previously measured hs-cTnT levels correlating them to a well-known marker, i.e. CRP, representing an index of acute phase reaction or ongoing inflammation in patients with NAFLD on maintenance haemodialysis.

Furthermore, we sought any relation between hs-cTnT levels and albumin concentrations as well as body composition indices.

\section{METHODS}

\section{Study Design}

This is a preliminary retrospective study, whose data were collected from patients' records and influenced in no way the decision-making of physicians, who strictly followed the diagnosis and therapy guidelines in caring for their haemodialysed patients. For this type of study formal consent is not required and ethics committee approval is unnecessary. Data is deidentified and one author, affiliated to the dialysis centre, had permission to use/access the database.

\section{Population}

We evaluated data from 70 patients with ESRD out of 170, in the period from 2015 to 2019 . The patients with ESRD were undergoing haemodialysis treatment, suffered from NAFLD, diagnosed by ultrasonography, but not for at least 3 months from symptomatic angina and had no history of ischemic heart disease in the same period. We excluded patients whose abdominal ultrasonography was not recorded, even though many of them suffered from type 2 diabetes mellitus and hypertension.

Furthermore, patients with acute syndromes such as sepsis (fever, leucocytosis/leucopenia), pulmonary embolism (D-dimer level) or myocarditis (echocardiography) were opportunely ruled out according to recommended diagnostic guidelines. 


\section{Laboratory Data}

Hs-cTnT levels were measured by electrochemiluminescence immunoassay (ECLIA, Roche Diagnostics). The limit of detection (LoD) of the assay was $5 \mathrm{ng} / \mathrm{L}$ and the interassay imprecision was less than $10 \%$ [20].

Measurement of CRP was performed by Tinaquant C-Reactive Protein Gen.3 (Roche Diagnostics $\mathrm{GmbH})$. The LoD of the assay was $0.6 \mathrm{mg} / \mathrm{L}$ and the interassay imprecision was less than $7.0 \%$ [21].

As the replacement therapy was performed 3 days/week, the levels of both hs-cTnT and CRP were detected, as random values, at the same time at the end of the longest interval, just before starting the treatment, after a period of dialysis amounting to at least six a month. The determination was performed just before the haemodialysis in order to avoid the potential removal by the treatment.

\section{Body Composition Parameters}

Fat mass (FM) and free fat mass (FFM) were measured as percentage by a bioimpedance spectroscopy technique. It measures the phase angle at 50 frequencies over a range from 5 to $1000 \mathrm{kHz}$ to determine the electrical resistance of the total body water and of the extracellular water, using the BCM-Body Composition Monitor assessment of total body water by Fresenius Medical Care, Hong Kong. This method is validated against gold standard reference methods (deuterium dilution).

\section{Echocardiography}

Echocardiography (GE Vivid 7 Dimension ${ }^{\circledR}$ ultrasound system, General Electric Company, Fairfield, Connecticut, USA) was performed on 39 patients (27 men and 12 women) to study the ejection fraction (EF), the most important and influential factor for dichotomising patients with heart failure (HF) into the current classification of either preserved EF (HFpEF) or reduced $\mathrm{EF}(\mathrm{HFrEF}<40 \%)$ forms [22].
Echocardiography and bioimpedance analysis were performed in a period very close to hscTnT and CRP determinations.

\section{Statistics}

Variables not normally distributed, assessed by the Shapiro-Wilk W test (hs-cTnT CRP, albumin, FFM and FM), were expressed as median plus 25 th to 75 th interquartile range (IQR). Age and $\mathrm{EF}$, normally distributed, were expressed as mean \pm SD.

Two-sample Wilcoxon rank-sum (Mann-Whitney) test was set to evaluate differences in the same population according to gender.

For nonparametric tests of hypotheses Spearman's rho correlation was used to assess basal relationships.

To assess correlations, in univariate analysis, the linear regression analysis (ordinary least squares) was used, evaluating the coefficient, standard error, $t$ value, $P, 95 \%$ confidence intervals (CI) and $R^{2}$. When heteroscedasticity was suspected, i.e. when there were subpopulations that have different variabilities from others in the homoscedastic model, and having detected the presence of few outliers, we analysed the correlation by robust regression using least absolute deviations (LAD) regression.

For multivariate analysis, a robust multiple regression model was applied to evidence the eventual presence of co-factors or mediators in predicting the dependent variable. In this model, standardised coefficients or beta values were also calculated in order to compare different predictors to see which one was more important.

\section{RESULTS}

Twenty-seven women (38.5\%) and 43 men $(61.5 \%)$ formed our population, whose mean \pm SD of age was $69.1 \pm 13.7$ years. The clinical characteristics of haemodialysed patients are summarised in Table 1 . The median values of hs-cTnT, CRP, albumin, FM and FFM were $\quad 66.0 \mathrm{ng} / \mathrm{L} \quad(\mathrm{IQR}=42-105), \quad 6.85 \mathrm{mg} / \mathrm{L}$ $(\mathrm{IQR}=2.7-20.2), \quad 3.63 \mathrm{~g} / \mathrm{L} \quad(\mathrm{IQR}=3.63-3.88)$, 
Table 1 Clinical characteristics of the haemodialysed patients

\begin{tabular}{lll}
\hline Previous morbidities & $\begin{array}{l}\text { Number of } \\
\text { patients }(\%)\end{array}$ & Comorbidity \\
\hline $\begin{array}{l}\text { Type 2 diabetes } \\
\text { mellitus }\end{array}$ & $21(30.0)$ & NAFLD \\
$\begin{array}{l}\text { Glomerulonephritis } \\
\text { on steroids }\end{array}$ & $8(11.4)$ & NAFLD \\
Hypertension & $7(10.0)$ & NAFLD \\
$\begin{array}{l}\text { Interstitial nephritis } \\
\text { Pyelonephritis }\end{array}$ & $3(4.3)$ & NAFLD \\
Cancers & $2(2.9)$ & NAFLD \\
$\begin{array}{l}\text { Nephrocalcinosis } \\
\text { HCV-related chronic } \\
\text { disease }\end{array}$ & $1(1.4)$ & NAFLD \\
Undetermined & $20(28.6)$ & NAFLD \\
aetiology & & NAFLD \\
\hline
\end{tabular}

NAFDL nonalcoholic fatty liver disease detected at ultrasound

$14.6 \mathrm{~kg} / \mathrm{m}^{2}(\mathrm{IQR}=10.9-18.2)$ and $11.4 \mathrm{~kg} / \mathrm{m}^{2}$ (9.5-13), respectively. The mean \pm SD EF of 39 patients on echocardiography was $54.7 \pm 6.1 \%$. The results of these variables according to gender are shown in Table 2. There were differences only in troponin levels and FFM between men and women: $z=-1.98$; Prob $>|z|=0.047$ and $z=-2.95$; Prob $>|z|=0.0032$, respectively.

\section{Relationships/Correlations}

First of all, hs-cTnT levels and CRP values were correlated (rho $=0.34, P=0.004$ ), as were hscTnT levels and age (rho $=0.47, P=0.0001$ ). The relatively low value of rho, although significant, regarding the correlation between hscTnT and CRP levels could be due to the small sample size.

Serum concentrations of hs-cTnT for the whole population were positively predicted by CRP levels in robust regression analysis: coefficient $=2.32$, standard error $=0.78, t=2.98$, $P=0.004, \quad 95 \% \quad \mathrm{CI}=0.77-3.87, \quad R^{2}=0.28$ (Fig. 1).

On separation of the population by gender, no significant correlation was found between hs-cTnT and CRP among women $(n=23$ out of $70)$, but one was present in men: coefficient $=0.19$, standard error $=0.44, \quad t=0.44$, $P=0.66, \quad \mathrm{CI}=-0.71$ to 1.10 and coefficient $=3.36$, standard error $=0.62, t=5.40$, $P=0.000, \quad 95 \% \quad \mathrm{CI}=-4.62, \quad$ respectively, $R^{2}=0.28$ (Fig. 2).

There was a clearly significant correlation between age and hs-cTnT levels: coefficient $=2.53$, standard error $=0.75, t=3.40$, $P=0.001, \quad 95 \% \quad \mathrm{CI}=1.04-4.02, \quad R^{2}=0.15$ (Fig. 3).

On multiple regression using a combination of age and CRP levels and selecting only the male population, age was no longer significantly correlated with hs-cTnT levels while CRP maintained its predictability: $\mathrm{CRP}-$ coefficient $=3.10, \quad$ standard error $=0.55, \quad t=5.53$, $P=0.0001, \quad 95 \% \quad \mathrm{CI}=1.94-4.18 ; \quad$ age-

Table 2 Values of the parameters according to gender

\begin{tabular}{lllll}
\hline Parameters & Men & Women & $\boldsymbol{Z}$ & $\boldsymbol{P}$ \\
\hline hs-cTnT $(\mathrm{ng} / \mathrm{L})$ & $75.0(45.0-123.0)$ & $61.0(33.0-85.0)$ & -1.98 & 0.047 \\
CRP $(\mathrm{mg} / \mathrm{L})$ & $4.3(2.3-20.9)$ & $11.0(4.4-20.2)$ & 1.44 & 0.15 \\
Albumin $(\mathrm{g} / \mathrm{L})$ & $3.6(3.3-3.9)$ & $3.6(3.3-3.9)$ & -0.06 & 0.95 \\
FFM $\left(\mathrm{kg} / \mathrm{m}^{2}\right)$ & $12.2(9.8-13.7)$ & $10.2(8.5-11.8)$ & -2.95 & 0.003 \\
FM $\left(\mathrm{kg} / \mathrm{m}^{2}\right)$ & $13.8(10.8-16.6)$ & $17.1(11.4-20.3)$ & 1.42 & 0.15 \\
EF $(\%)$ & $54.1 \pm 6.4$ & $56.2 \pm 5.2$ & 0.71 & 0.47 \\
\hline
\end{tabular}

bs-cTnT high sensitivity cardiac troponin, $C R P$ C-reactive protein, $F F M$ free fat mass, $F M$ fat mass, $E F$ ejection fraction 


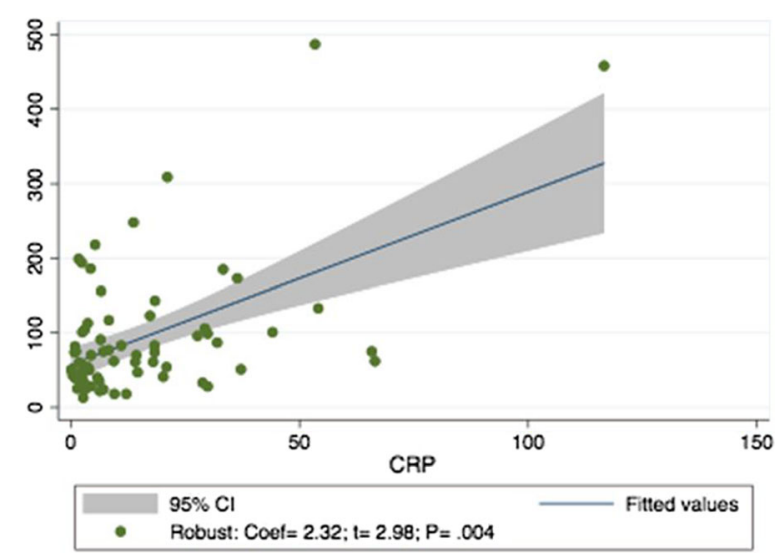

Fig. 1 Linear regression, CRP levels expressed as mg/L; hscTnT levels shown as ng/L

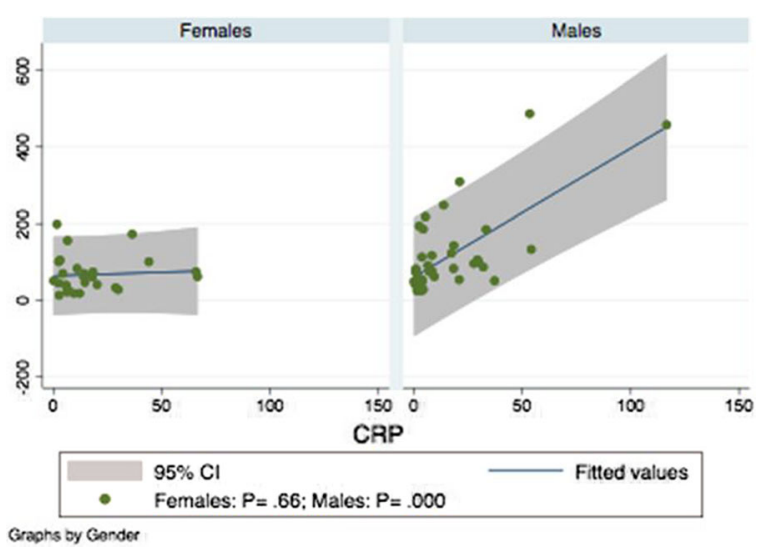

Fig. 2 Linear regression, robust model. CRP levels expressed as $\mathrm{mg} / \mathrm{L}$; hs-cTnT levels shown as $\mathrm{ng} / \mathrm{L}$. It is noteworthy to stress that only two values are outside the CI lines in men $(n=47)$

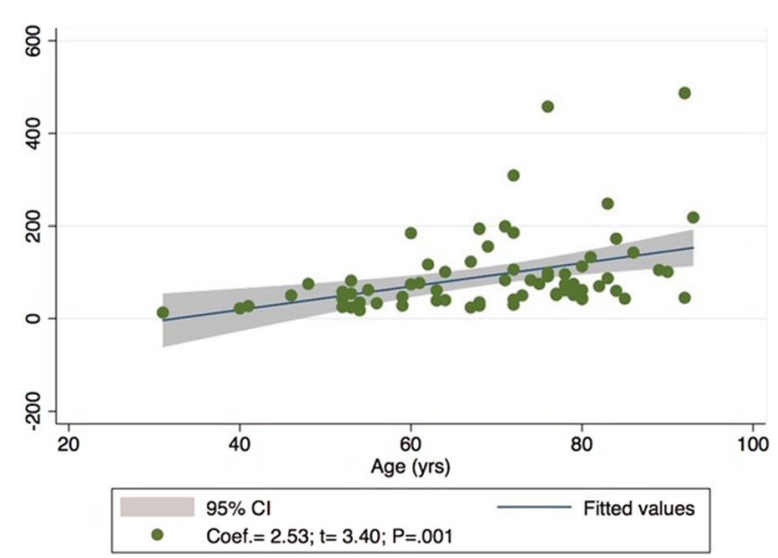

Fig. 3 Linear regression, robust model. CRP levels expressed as $\mathrm{mg} / \mathrm{L}$; age shown as years

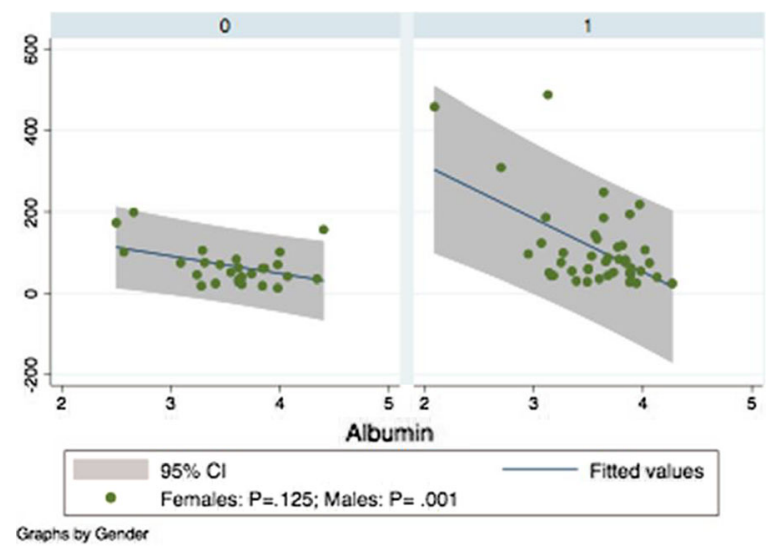

Fig. 4 Prediction of hs-cTnT by albumin; hs-cTnT levels were presented as $\mathrm{ng} / \mathrm{mL}$, albumin concentrations as $\mathrm{g} / \mathrm{L}$, left panel $=$ women $(n=27)$ and right panel $=$ men $(n=43)$

coefficient $=1.44, \quad$ standard $\quad$ error $=0.84$, $t=1.71, P=0.096,95 \% \mathrm{CI}=-0.27$ to 3.15 . The beta values for CRP and age were 45.70 and 27.80, respectively; $R^{2}=0.35$.

When predicting the behaviour of troponin using as albumin concentration as the independent variable according to gender, we obtained an interesting result-albumin concentrations did not predict hs-cTnT levels in women: coefficient $=-43.05$, standard error $=$ 27.14, $t=-1.59, P=0.125,95 \% \mathrm{CI}=-98.94$ to $12.85, R^{2}=0.19$; whereas hs-cTnT concentrations were predicted by albumin levels in men: coefficient $=-131.64$, standard error $=$ 43.55, $\quad t=-3.02, \quad P=0.001, \quad 95 \%$ $\mathrm{CI}=-219.58$ to $43.69, R^{2}=0.28$ (Fig. 4 ).

Both FM and FFM failed to predict hs-cTnT levels, including when they were adjusted for gender (women: $P=0.9$ and 0.26 ; men: $P=0.61$ and 0.06).

On robust regression, neither hs-cTnT levels nor CRP concentrations were correlated with the duration of dialysis, expressed in months of given treatment: coefficient $=0.12$, standard error $=0.20, t=0.61, P=0.55, \mathrm{CI}=-0.28$ to 0.53 and coefficient $=0.61$, standard error $=$ $0.41, t=1.49, P=0.14 \mathrm{CI}=-0.20$ to 0.14 , respectively. For 39 patients with HFpEF, hscTnT serum concentrations were not predicted by CRP levels on robust regression: coefficient $=2.72$, standard error $=1.83, t=1.49$, 
$P=0.145, \quad 95 \% \quad \mathrm{CI}=23.04-6.433, \quad R^{2}=0.23$. Similarly, EF was not predicted by hs-cTnT levels: coefficient $=-0.033$, standard error $=$ $0.11, t=-0.29, P=0.77, \mathrm{CI}=-0.26$ to 0.19 , $R^{2}=0.002$, respectively.

\section{DISCUSSION}

As previously emphasised, in patients with ESRD, serum troponin concentrations tend to be higher than in the general population even in the absence of acute events. Recently, many authors have dedicated themselves to the evaluation of the prognostic significance of cardiac troponin levels in patients with ESRD [23]. Moreover, it should be highlighted that CRP has been found elevated in patients with NAFLD [24]. In this regard, evidence linking NAFLD to the development of CKD, and then ESRD, is emerging as a popular area of scientific interest [25].

In our study we compared hs-cTnT levels with varying degrees of inflammation or cardiovascular risk by a recognised marker, i.e. CRP.

As core finding we showed that hs-cTnT concentrations in our population of haemodialysed patients with NAFLD positively correlated with CRP levels. However, little is known regarding the potential complementary roles of measuring levels of cTnT and CRP, nor the underlying cardiac pathology in stable patients with ESRD and whether these biomarkers are indicators of the severity of either coronary atherosclerosis demonstrated by angiography or of cardiomyopathy as manifested by left ventricular systolic dysfunction or hypertrophy determined by echocardiography [5].

Indeed, the strong association between hscTnT and CRP lends credence to the presence of possible myocardial damage, as a reflection of inflammation within the arterial walls concentrated in some specific areas (a vulnerable plaque) [26]. Trying to give an explanation about the mechanisms, we hypothesise that the hs-cTnT increase has a myocardial origin, due to cardiac muscle release, even though there was no contextual collection of instrumental data, i.e. ST segment changes consistent with ischemia seen during exercise treadmill testing or ambulatory monitoring, or reversible myocardial perfusion defects noted during radionuclide myocardial perfusion imaging. Specifically, the link between ESRD and NAFLD could be represented by oxidative stress, as evidenced by research evaluating an oxidative stress marker (GPx activity) and mitochondrial DNA copy number [27].

\section{Implications of These Results}

It has been seen that patients with ESRD, under dialysis or not, with higher values of troponin have a worse prognosis; in particular, their risk of cardiovascular mortality and major cardiac adverse events was 2-4 times greater than those with lower cardiac troponin values [28]. Moreover, it has been shown that elevated levels of inflammatory biomarkers, including CRP, are associated with an increased risk of death from all causes. Furthermore, compared with those in the lowest quartile of hs-cTnT, patients in the highest quartile demonstrated a nearly fivefold higher risk of cardiovascular death and heart failure hospitalisation with a hazard ratio of 4.85 [29]. The lack of prediction of hs-cTnT levels by CRP in patients with HFpEF confirms this aspect as well the absent prediction of EF by hs-cTnT levels [30].

Contrasting our results with literature data, we stress the following: Our study was based on records concerning clinical, laboratory and instrumental data gathered in a close time period. It showed that in patients undergoing haemodialysis the gender played a pivotal role in predicting hs-cTnT levels, and for this aspect it is novel. Previous research in patients with NAFLD emphasised the role of male gender, which was associated with a major risk of $\mathrm{CV}$ events [31], but our findings linked this aspect to a specific marker, such as troponin. Furthermore, the correlation of age and hs-cTnT in our population of dialysed patients, characterised by advanced age, confirms recent research in 679 elderly hospitalised patients. This analysis found that age is independently associated with risk of increased cTnT as well as male gender. Another study found that local inflammation increased with age in plaques obtained from patients, especially men, suffering from haemodynamically significant advanced 
atherosclerotic lesions [32]. Clearly, the presence of chronic disease and the aging process itself pose particular challenges when using hscTnT to diagnose ACS in the elderly. This prompts nephrologists to take into account the possibility that hs-cTnT increase is due to renal dysfunction (reduced eGFR) [33, 34]. But, these findings do not exclude that troponin elevation could have cardiac origin.

Although no difference between albumin serum concentrations in men and women was found, the albumin levels of men nevertheless predicted hs-cTnT concentrations. Possible hypotheses for this discrepancy could consist in concomitant liver disease or in somehow concomitant heart dysfunction [35]. The speculation that albumin concentrations were linked to nutritional status deficiency was not confirmed in our population because hs-cTnT was not predicted by FFM and FM in both genders. At this point it is noteworthy that in 39 patients with HFpEF, hs-cTnT serum concentrations were not predicted by CRP levels, nor was EF predicted by hs-cTnT levels.

In summary, the assessment of troponin levels could represent a biological marker to identify those most at risk of major adverse cardiac events in haemodialysis patients, especially in particular subgroups such as for male patients with higher CRP, and then to indicate which of them deserve stricter surveillance and more aggressive therapy with beta blockers and/ or ACE inhibitors. This major risk is likely due to SMI that can occur in the absence of chest discomfort or other angina-equivalent symptoms, e.g. dyspnoea, nausea, diaphoresis, etc., with ST segment changes on ECG, reversible regional wall motion abnormalities, or perfusion defects on scintigraphy studies [36]. Another potential event is the presence of asymptomatic arrhythmias including atrial fibrillation [37]. Our patients undergoing haemodialysis experienced a nearly 5\% incidence of atrial fibrillation.

\section{Limitations}

The specific selection of patients could have influenced the results, but it was our aim to verify the behaviour of troponin and CRP in a particular subgroup of dialysed patients.

Although the retrospective nature of our study does not permit definitive conclusions because of its intrinsic lack of data accuracy, it opens the door to new studies. Furthermore, we point out the relatively small size of our population that could have had a limited impact on statistical analysis.

\section{Future Directions}

It could be interesting to evaluate in a prospective study the incidence of adverse cardiovascular events and all-cause mortality over time in patients with NAFLD and higher hscTnT levels according to the above hypothesis. Current monitoring of serum troponin levels in the most inflamed patients could also be a way to verify the effect of implemented therapeutic strategies. It is noteworthy that many haemodialysed patients are not prescribed cardioprotective medications [38]. Prospective long-term studies are needed to deepen the content of this research.

\section{CONCLUSION}

The assessment of troponin levels in haemodialysis patients could be useful to identify, in male patients with higher CRP, those at greater risk of SMI and future major adverse cardiac events, thus indicating which of them should undergo monitoring and adopt specific therapy, considering that this preliminary research could be important to set up further studies.

\section{ACKNOWLEDGEMENTS}

Funding. No funding or sponsorship was received for this study or publication of this article.

Authorship. All named authors meet the International Committee of Medical Journal 
Editors (ICMJE) criteria for authorship for this article, take responsibility for the integrity of the work as a whole, and have given their approval for this version to be published.

Disclosures. Domenico Capone is no longer affiliated to Federico II University Medical School of Naples, Italy; currently this author is a Clinical Pharmacology Consultant and has nothing else to disclose. Giovanni Tarantino is a section Editor for Advances in Therapy but has nothing further to disclose. The remaining authors Domenico Capone, Mauro Vinciguerra, Annalisa Ragosta and Vincenzo Citro have nothing to disclose.

Compliance with Ethics Guidelines. For this type of study formal consent is not required and ethics committee approval is unnecessary. Data is de-identified and one author, affiliated to the dialysis centre, had permission to use/ access the database.

Data Availability. The datasets generated during and/or analysed during the current study are available from the corresponding author on reasonable request.

Open Access. This article is licensed under a Creative Commons Attribution-NonCommercial 4.0 International License, which permits any non-commercial use, sharing, adaptation, distribution and reproduction in any medium or format, as long as you give appropriate credit to the original author(s) and the source, provide a link to the Creative Commons licence, and indicate if changes were made. The images or other third party material in this article are included in the article's Creative Commons licence, unless indicated otherwise in a credit line to the material. If material is not included in the article's Creative Commons licence and your intended use is not permitted by statutory regulation or exceeds the permitted use, you will need to obtain permission directly from the copyright holder. To view a copy of this licence, visit http://creativecommons.org/licenses/by$\mathrm{nc} / 4.0 /$.

\section{REFERENCES}

1. Dawwas GK, Liu X, Nguyen MH. Nonalcoholic fatty liver disease increases risk of incident advanced chronic kidney disease: a propensity-matched cohort study. J Intern Med. 2019;286:711-22.

2. Wu S, Wu F, Ding Y, Hou J, Bi J, Zhang Z. Association of non-alcoholic fatty liver disease with major adverse cardiovascular events: a systematic review and meta-analysis. Sci Rep. 2016;6:33386.

3. Chinnadurai R, Ritchie J, Green D, Kalra PA. Nonalcoholic fatty liver disease and clinical outcomes in chronic kidney disease. Nephrol Dial Transplant. 2019;34:449-57.

4. Mikolasevic I, Racki S, Zaputovic L, Lukenda V, Sladoje-Martinovic B, Orlic L. Nonalcoholic fatty liver disease (NAFLD) and cardiovascular risk in renal transplant recipients. Kidney Blood Press Res. 2014;39:308-14.

5. de Filippi C, Wasserman S, Rosanio S, et al. Cardiac troponin $\mathrm{T}$ and $\mathrm{C}$-reactive protein for predicting prognosis. Coronary atherosclerosis, and cardiomyopathy in patients undergoing long-term hemodialysis. JAMA. 2003;290:353-9.

6. Michos ED, Wilson LM, Yeh HC, et al. Prognostic value of cardiac troponin in patients with chronic kidney disease without suspected acute coronary syndrome: a systematic review and meta-analysis. Ann Intern Med. 2014;161:491-501.

7. Castini D, Persampieri S, Floreani R, et al. Troponin I levels in asymptomatic hemodialysis patients. Blood Purif. 2017;44:236-43.

8. Fox WR, Diercks DB. Troponin assay use in the emergency department for management of patients with potential acute coronary syndrome: current use and future directions. Clin Exp Emerg Med. 2016;3:1-8.

9. Skeik N, Patel DC. A review of troponins in ischemic heart disease and other conditions. Int J Angiol. 2007;16:53-8.

10. Chen M, Gerson H, Eintracht S, Nessim SJ, MacNamara E. Effect of hemodialysis on levels of high-sensitivity cardiac troponin T. Am J Cardiol. 2017;120:2061-4.

11. Narula AS, Jha V, Bali HK, Sakhuja V, Sapru RP. Cardiac arrhythmias and silent myocardial ischemia during hemodialysis. Ren Fail. 2000;22:355-68.

12. McIntyre CW. Recurrent circulatory stress: the dark side of dialysis. Semin Dial. 2010;23:449-51. 
13. Singh AT, Mc Causland FR. Osmolality and blood pressure stability during hemodialysis. Semin Dial. 2017;30:509-17.

14. Bucharles SGE, Wallbach KKS, Moraes TP, PecoitsFilho R. Hypertension in patients on dialysis: diagnosis, mechanisms, and management. J Bras Nefrol. 2019;4:400-11.

15. Kjeldsen SE. Hypertension and cardiovascular risk: general aspects. Pharmacol Res. 2018;129:95-9.

16. Zebrack JS, Anderson JL, Maycock CA, Horne BD, Bair TL, Muhlestein JB. Intermountain Heart Collaborative (IHC) Study. Group Usefulness of highsensitivity $\mathrm{C}$-reactive protein in predicting longterm risk of death or acute myocardial infarction in patients with unstable or stable angina pectoris or acute myocardial infarction. Am J Cardiol. 2002;89: 145-9.

17. Bagheri N, Taziki O, Falaknazi K. C-reactive protein, cardiac troponin $\mathrm{T}$ and low albumin are predictors of mortality in hemodialysis patients. Saudi J Kidney Dis Transpl. 2009;20:789-93.

18. Heidari B. C-reactive protein and other markers of inflammation in hemodialysis patients. Caspian J Intern Med. 2013;4:611-6.

19. de Lemos JA, Drazner MH, Omland T, et al. Association of troponin $\mathrm{T}$ detected with a highly sensitive assay and cardiac structure and mortality risk in the general population. JAMA. 2010;304:2503-12.

20. Giannitsis E, Kurz K, Hallermayer K, Jarausch J, Jaffe AS, Katus HA. Analytical validation of a high-sensitivity cardiac troponin $\mathrm{T}$ assay. Clin Chem. 2010;56:254-61.

21. Eda S, Kaufmann J, Roos W, Pohl S. Development of a new microparticle-enhanced turbidimetric assay for C-reactive protein with superior features in analytical sensitivity and dynamic range. J Clin Lab Anal. 1998;12:137-44.

22. Ponikowski P, Voors AA, Anker SD, et al. ESC Scientific Document Group. 2016 ESC Guidelines for the diagnosis and treatment of acute and chronic heart failure: The Task Force for the diagnosis and treatment of acute and chronic heart failure of the European Society of Cardiology (ESC)Developed with the special contribution of the Heart Failure Association (HFA) of the ESC. Eur Heart J. 2016;14: 2129-200.

23. Chen T, Hassan HC, Qian P, Vu M, Makris A. Highsensitivity troponin $\mathrm{T}$ and C-reactive protein have different prognostic values in hemo- and peritoneal dialysis populations: a cohort study. J Am Heart Assoc. 2018;7:e007876.
24. Yeniova AO, Küçükazman $\mathrm{M}$, Ata N, et al. Highsensitivity C-reactive protein is a strong predictor of non-alcoholic fatty liver disease. Hepatogastroenterology. 2016;61:422-5.

25. Marcuccilli M, Chonchol M. NAFLD and chronic kidney disease. Int J Mol Sci. 2016;17:562.

26. Griffiths ME, Malan L, Delport R, Cockeran M, Reimann M. Troponin T release is associated with silent myocardial ischaemia in black men: the SABPA Study. Eur J Prev Cardiol. 2017;24:942-50.

27. Wu PJ, Chen JB, Lee WC, et al. Oxidative stress and nonalcoholic fatty liver disease in hemodialysis patients. Biomed Res Int. 2018;1(2018):3961748.

28. Michos ED, Berger Z, Yeh HC, et al. Cardiac troponins used as diagnostic and prognostic tests in patients with kidney disease. Rockville: Agency for Healthcare Research and Quality (AHRQ). Comparative Effectiveness Review, No. 135; 2014.

29. Myhre PL, O'Meara E, Claggett BL, et al. Cardiac troponin I and risk of cardiac events in patients with heart failure and preserved ejection fraction. Circ Heart Fail. 2018;11:e005312.

30. Khan MH, Islam MN, Aditya GP, et al. Correlation of troponin-I level with left ventricular ejection fraction and in-hospital outcomes after first attack of non-ST segment elevation myocardial infarction. Mymensingh Med J. 2017;26:721-31.

31. Hagström H, Nasr P, Ekstedt M, et al. Cardiovascular risk factors in non-alcoholic fatty liver disease. Liver Int. 2019;39(1):197-204.

32. Hellings WE, Pasterkamp G, Verhoeven BA, et al. Gender-associated differences in plaque phenotype of patients undergoing carotid endarterectomy. J Vasc Surg. 2007;45:289-96.

33. Zhang S-J, Wang Q, Cui Y-J, et al. High-sensitivity cardiac troponin $\mathrm{T}$ in geriatric patients. Arch Gerontol Geriatr. 2016;56:111-5.

34. Dubin RF, Li Y, He J, et al. Predictors of high sensitivity cardiac troponin $\mathrm{T}$ in chronic kidney disease patients: a cross-sectional study in the chronic renal insufficiency cohort (CRIC). BMC Nephrol. 2013;14:229.

35. Forghani MS, Jadidoleslami MS, Naleini SN, Rajabnia M. Measurement of the serum levels of serum troponins I and $\mathrm{T}$, albumin and C-reactive protein in chronic hemodialysis patients and their relationship with left ventricular hypertrophy and heart failure. Diabetes Metab Syndr. 2019;13:522-5.

36. Cohn PF. Silent ischemia: a timely aspect in coronary artery disease. Herz. 1987;12:314-7. 
37. Kimura K, Tabei K, Asano Y, Hosoda S. Cardiac arrhythmias in hemodialysis patients. A study of incidence and contributory factors. Nephron. 1989;53:201-7.
38. Miller LM, Hopman WM, Garland JS, Yeates KE, Pilkey RM. Cardioprotective medication use in hemodialysis patients. Can J Cardiol. 2006;22: 755-60. 